
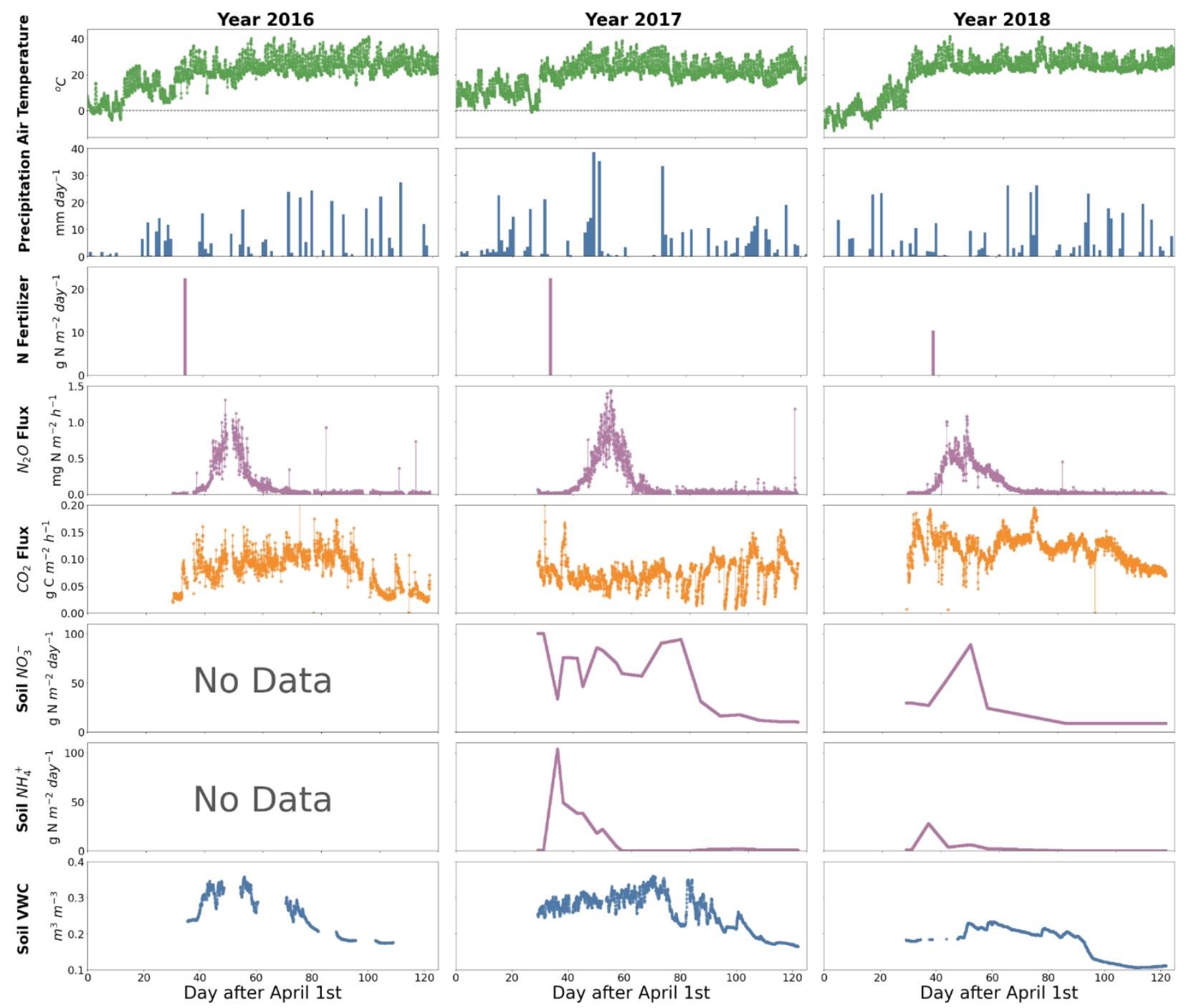

Figure S1: Time series example of observation data collected from mesocosm chamber 1. The precipitation, $\mathrm{N}$ fertilizer, $\mathrm{Soil}^{\mathrm{NO}_{3}}{ }^{-}$ and $\mathrm{NH}_{4}{ }^{+}$data are in daily time scale, while other data are in hourly time scale. Temperature presents in green; water related variables (precipitation and soil $\mathrm{VWC}$ ) are in blue; $\mathrm{N}$ related variables $\left(\mathrm{N}\right.$ fertilizer, $\mathrm{N}_{2} \mathrm{O}$ flux, $\mathrm{Soil} \mathrm{NO}_{3}{ }^{-}$and $\mathrm{NH}_{4}{ }^{+}$) are in purple;

5 and $\mathrm{CO}_{2}$ is in orange. Anomaly points will be down-weighted by daily averaging method with quality check in later processes, which is mentioned in section 2.2.2 last paragraph. 

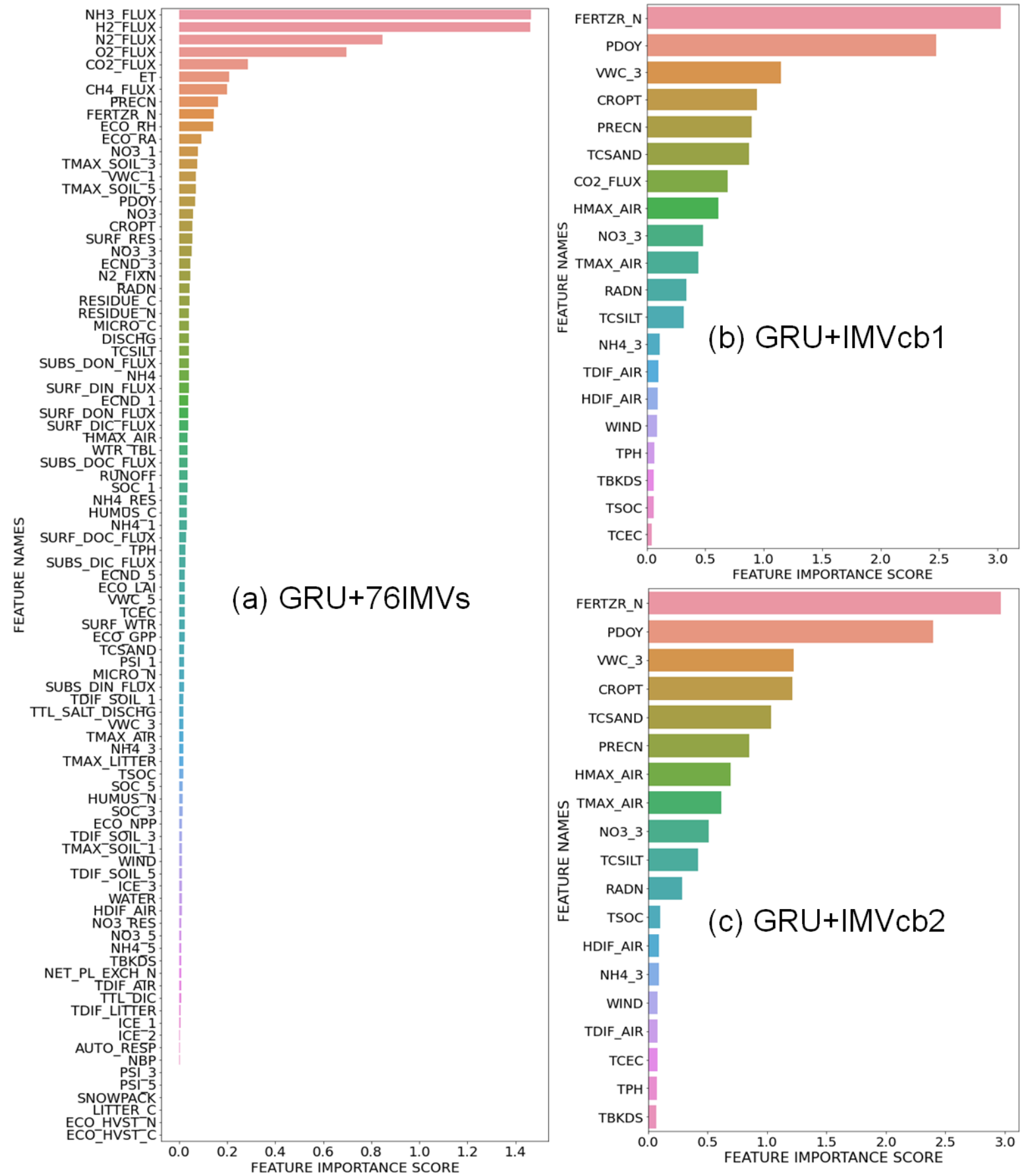

Figure S2: Feature importance test for intermediate variables (IMVs) with GRU models. To be noticed the $\mathrm{VWC} \mathrm{NO}_{3}{ }^{-}$, and $\mathrm{NH}_{4}{ }^{+}$

10 from third layer soil, which are presented in the main text are abbreviated here as $\mathrm{VWC}_{-} 3$, $\mathrm{NO}_{3} \_3$, and $\mathrm{NH}_{4} \_3$ to be distinguished from the same variables from 1st and 5th layers. Details of the variables can be found in Table S1. 


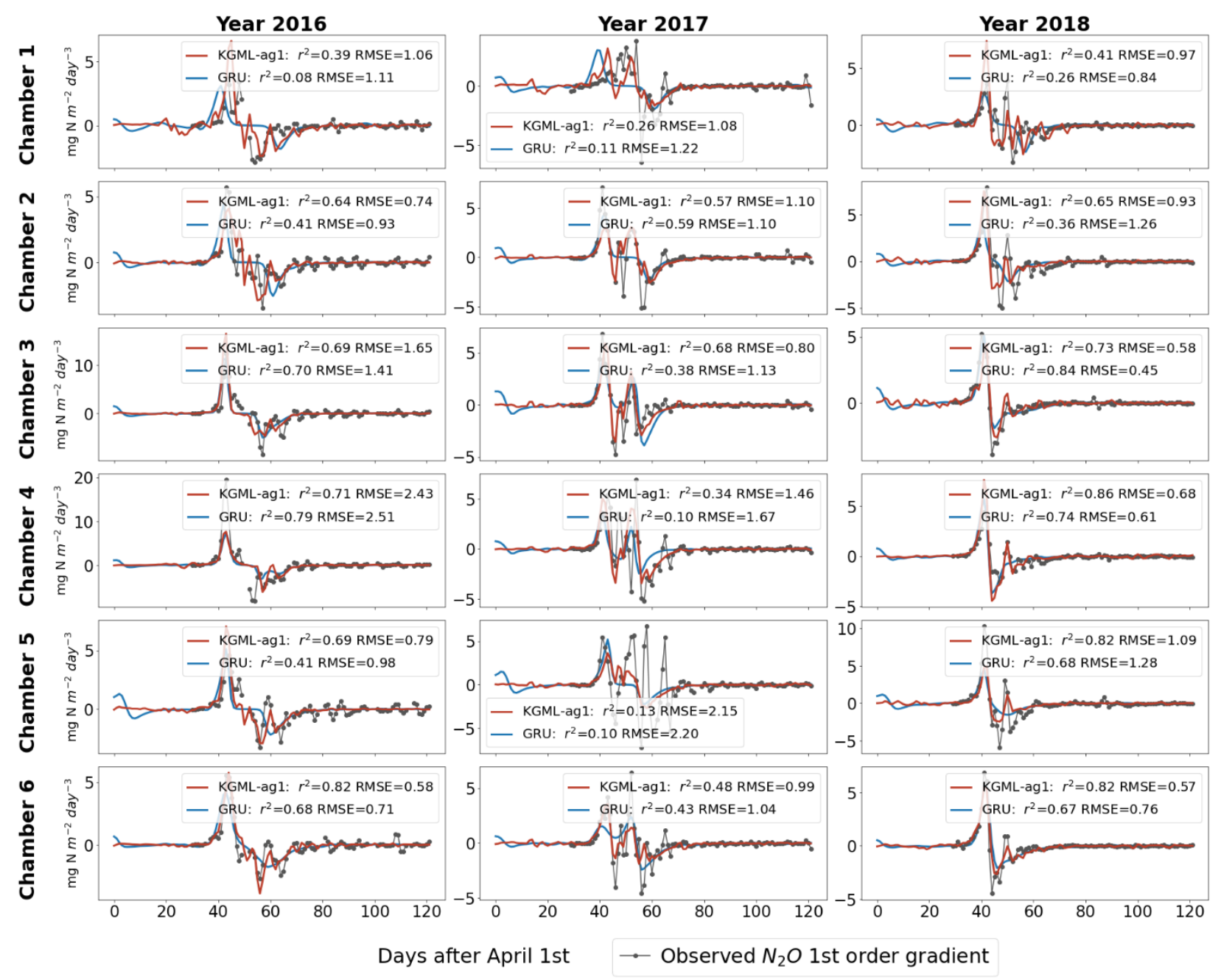

15 Figure S3: $\mathbf{N}_{2} \mathrm{O}$ flux 1st order gradient time series comparisons between non-pretrained GRU model and KGML-ag1. The blackdot line represents the observation, while blue represents GRU and red represents KGML-ag1. 

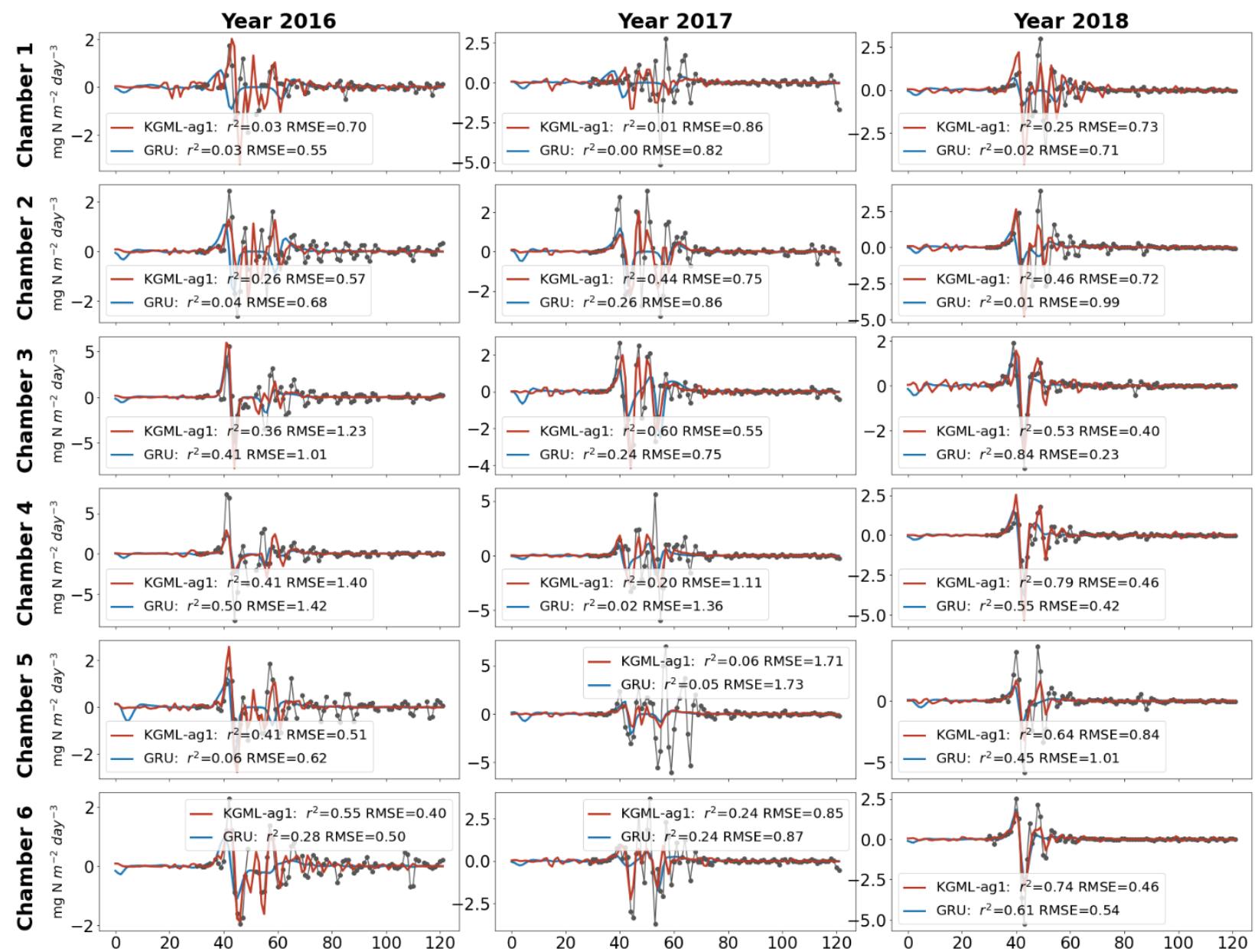

Days after April 1st

$\rightarrow$ Observed $\mathrm{N}_{2} \mathrm{O}$ 2nd order gradient

Figure S4: $\mathrm{N}_{2} \mathrm{O}$ flux 2nd order gradient time series comparisons between non-pretrained GRU model and KGML-ag1. The blackdot line represents the observation, while blue represents GRU and red represents KGML-ag1. 
(a) $\mathrm{CO}_{2}$ flux

Year 2016

Year 2017

Year 2018

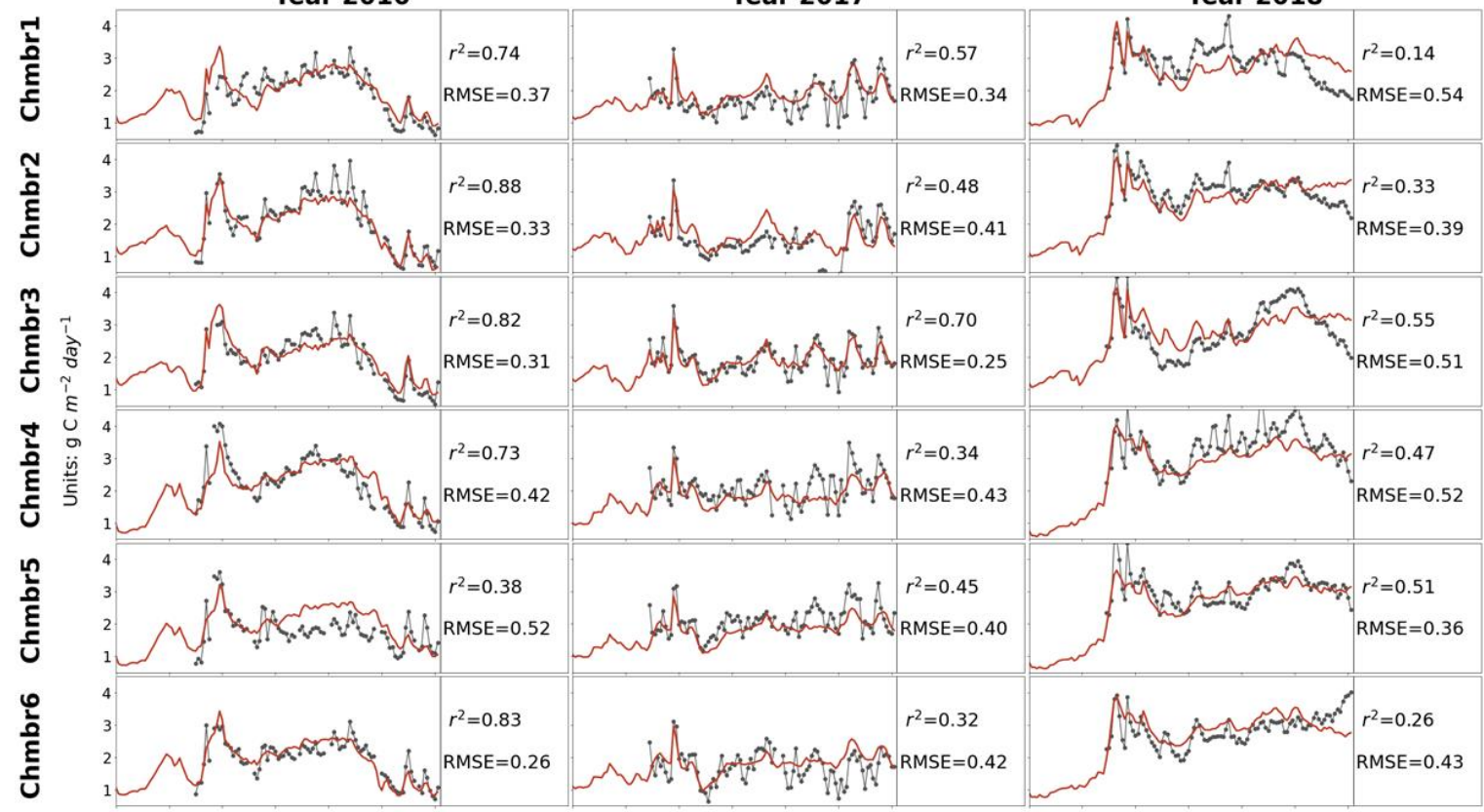

(b) soil $\mathrm{NO}_{3}^{-}$

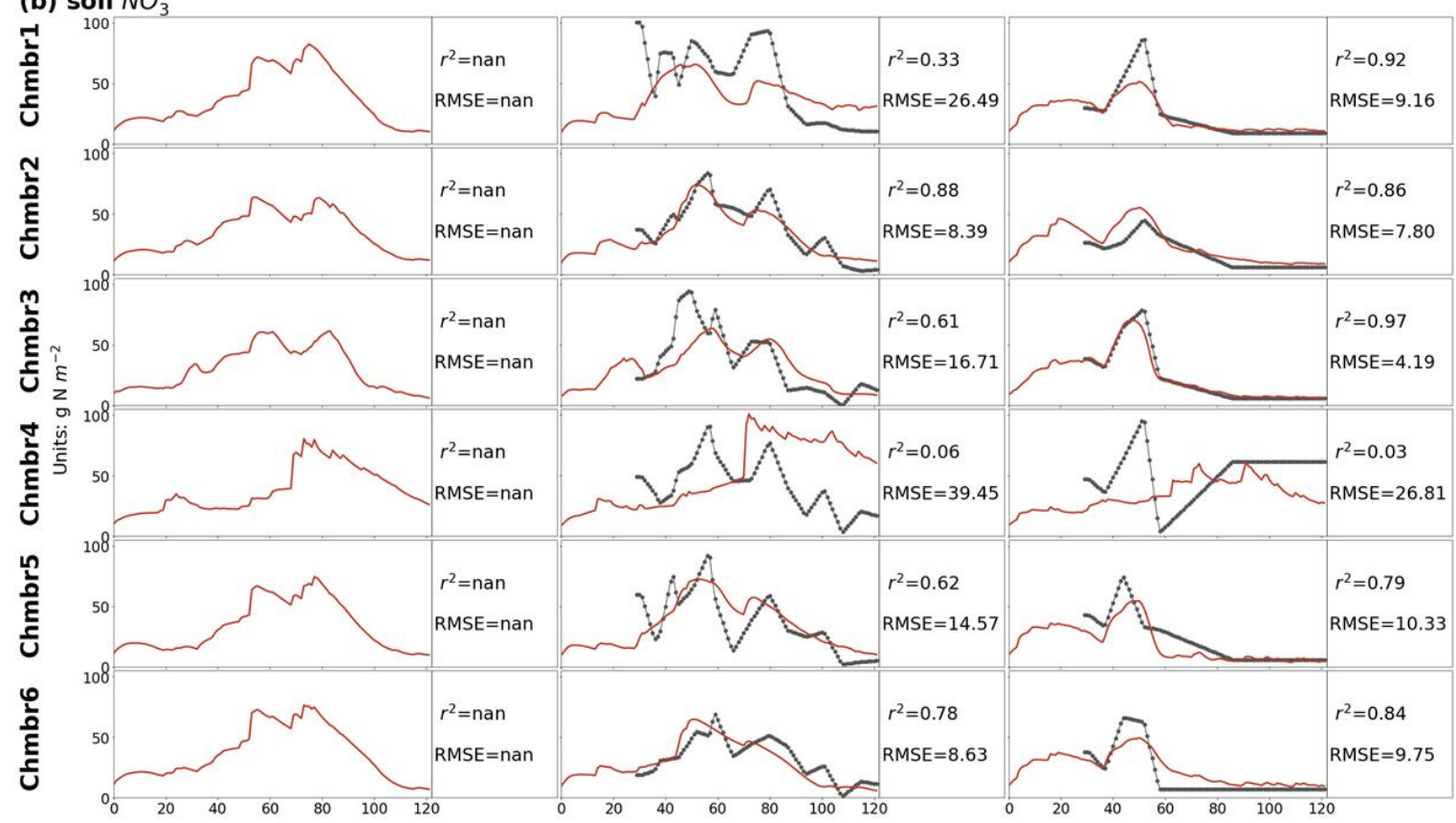

Days after April 1st $\quad$ KGML-ag2 $\rightarrow$ Observed Corresponding Variable

Figure S5: IMVs prediction from KGML-ag2. The black-dot line represents observations and the red line represents the results from KGML-ag2. Chmb is the abbreviation for chamber. $r^{2}$ and RMSE are calculated and present in each year and chamber. 
(c) soil $\mathrm{NH}_{4}^{+}$

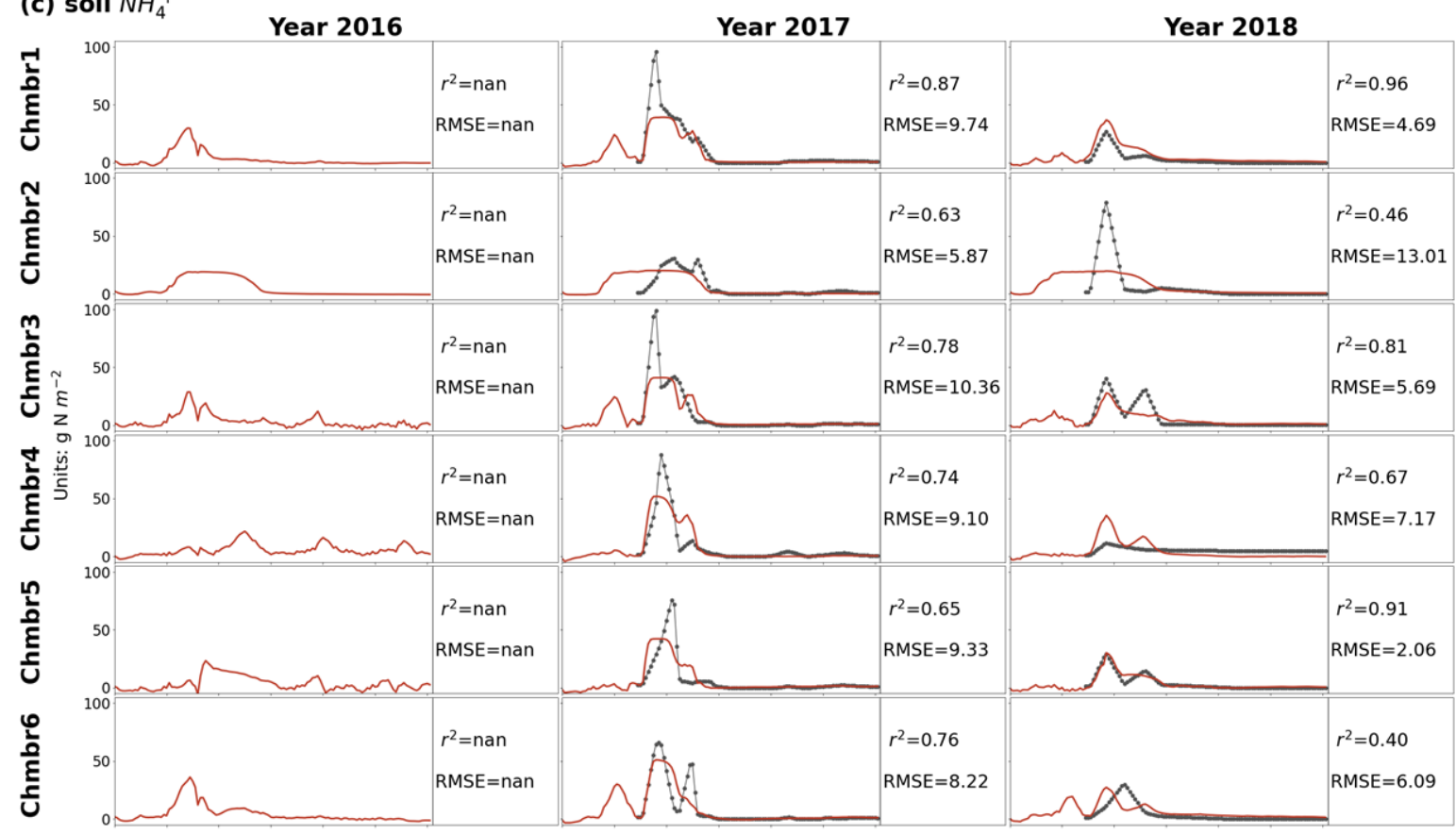

(d) soil VWC

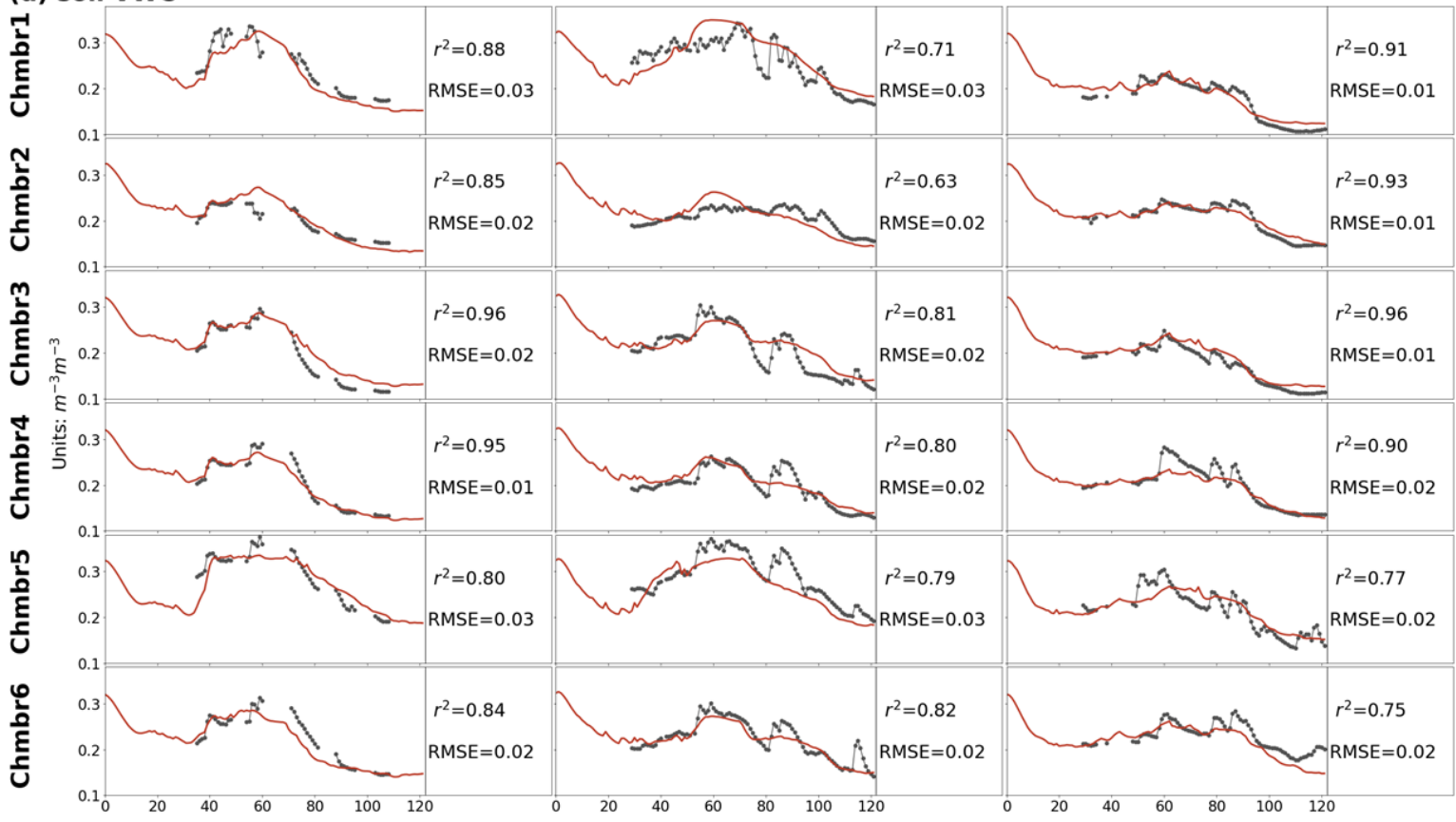

Days after April 1st $\quad$ KGML-ag2 $\rightarrow$ Observed Corresponding Variable

Figure S5 contd.: IMVs prediction from KGML-ag2. The black-dot line represents observations and the red line represents the results from KGML-ag2. Chmb is the abbreviation for chamber. $r^{2}$ and RMSE are calculated and present in each year and chamber. 
(a)

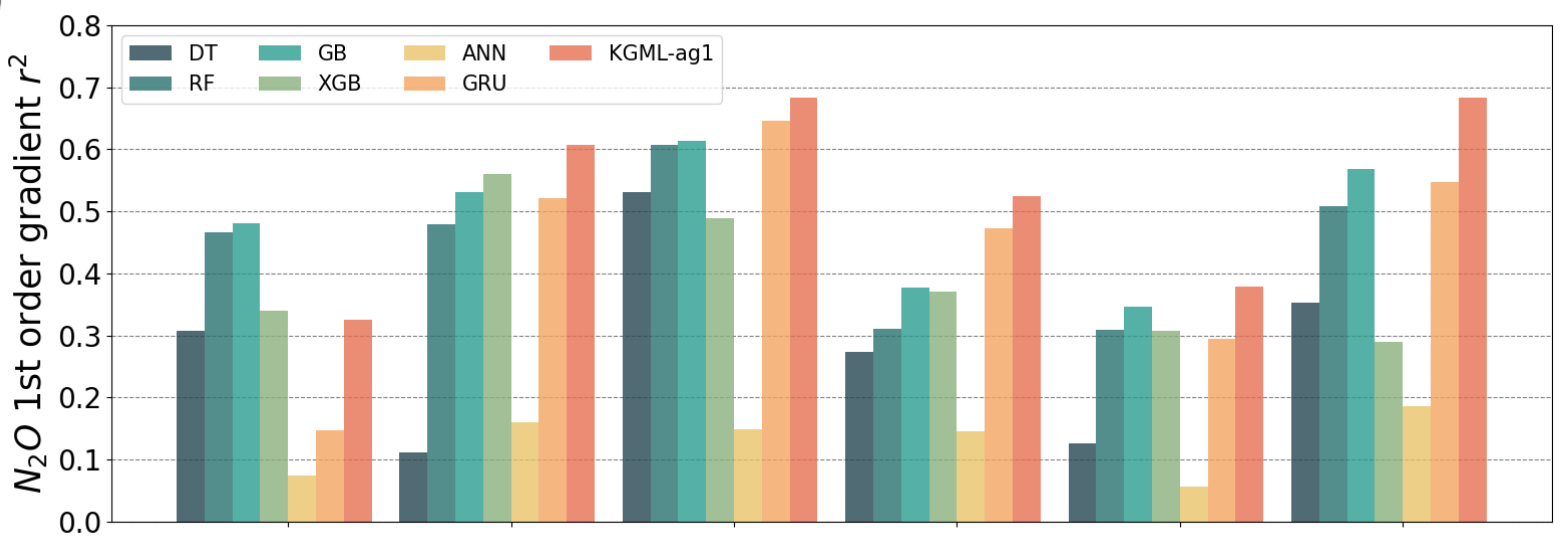

(b)

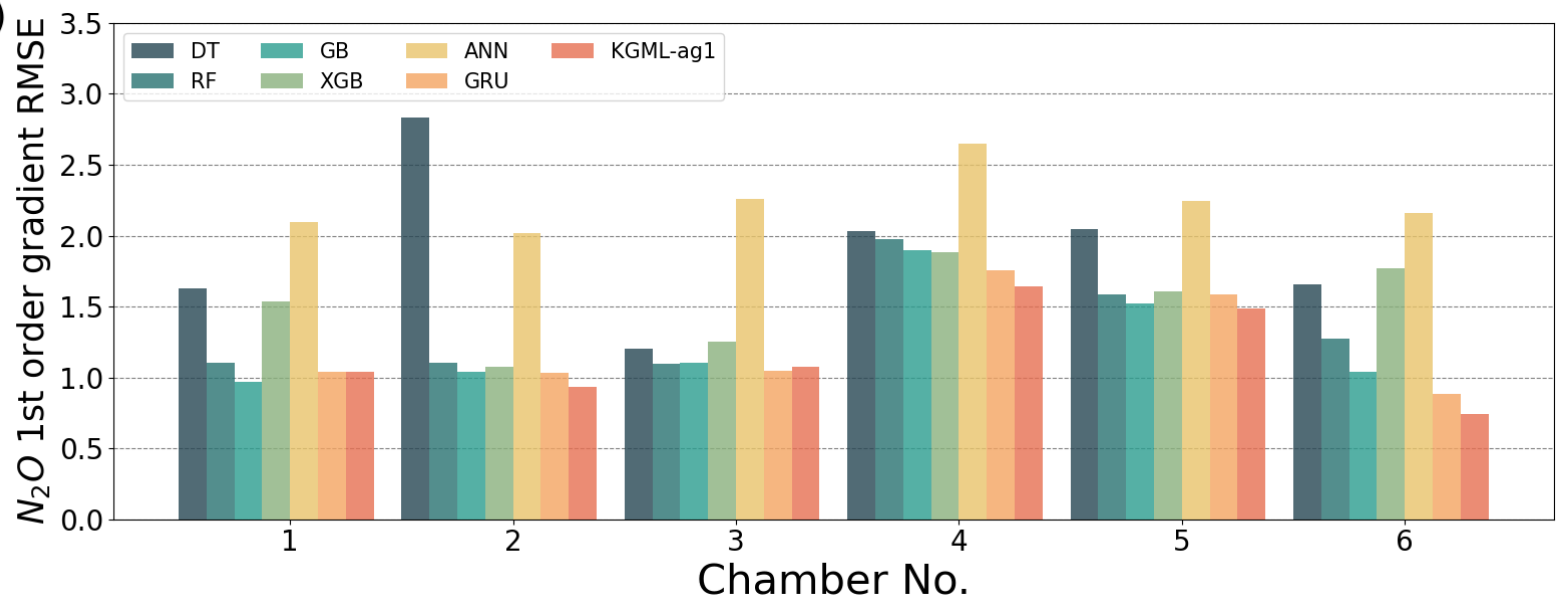

30 Figure S6: The comparisons of $\mathrm{N}_{2} \mathrm{O}$ 1st order gradient prediction accuracy $\mathbf{r}^{2}$ (a) and (b) RMSE, between four tree-based ML models (DT, RF, GB and XGB), two deep learning models (ANN and GRU) and KGML-ag1 model in 6 chambers. 
(a)

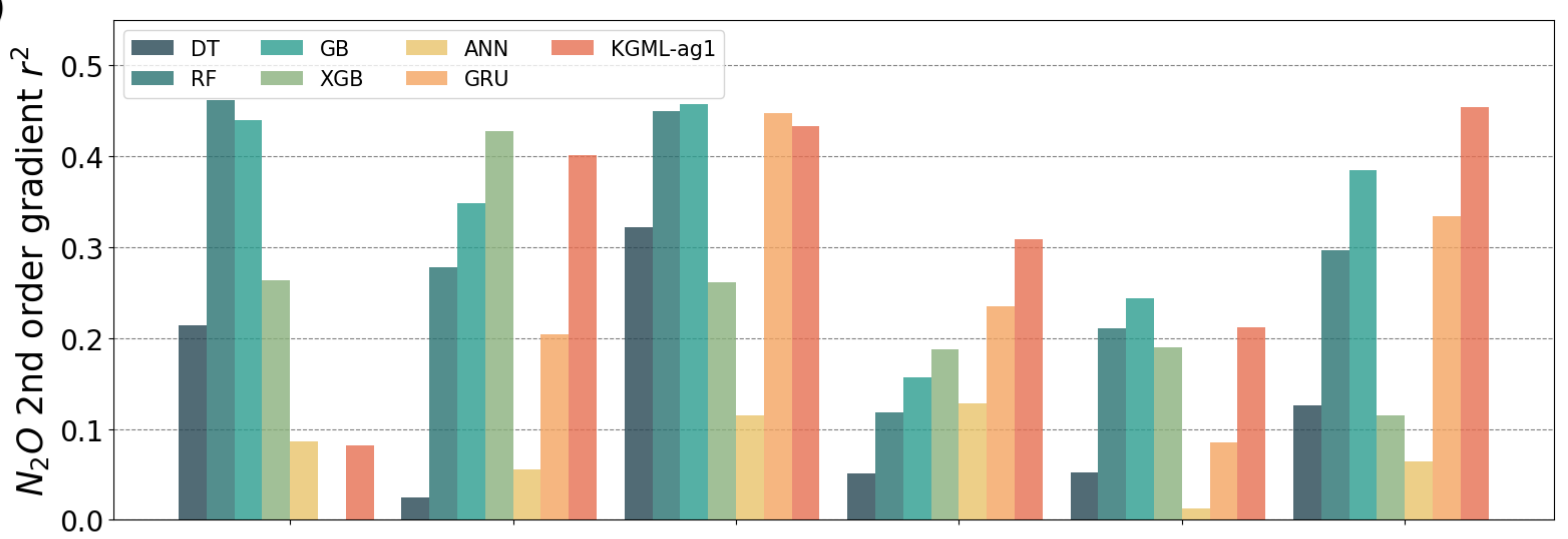

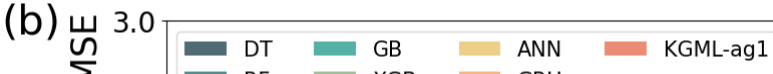

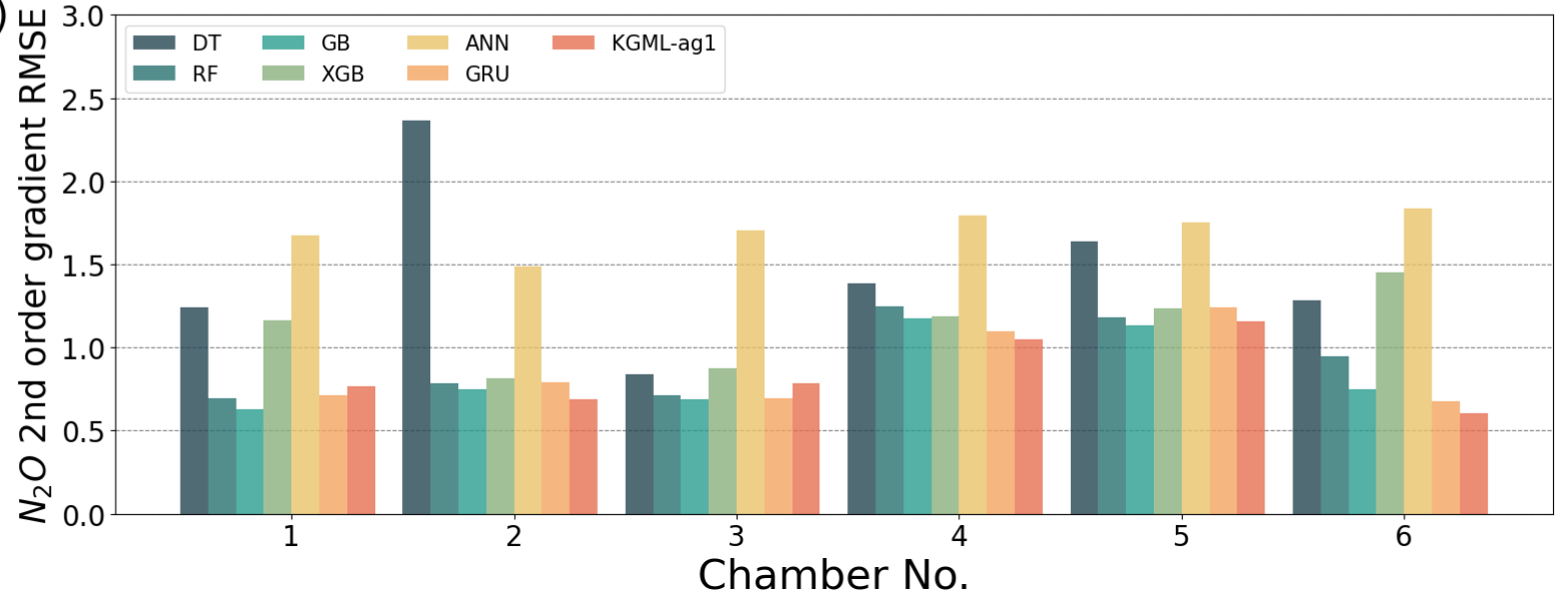

Figure S7: The comparisons of $\mathrm{N}_{2} \mathrm{O}$ 2nd order gradient prediction accuracy $\mathbf{r}^{2}(a)$ and (b) RMSE, between four tree-based ML models (DT, RF, GB and XGB), two deep learning models (ANN and GRU) and KGML-ag1 model in 6 chambers. 


\section{Year 2017}
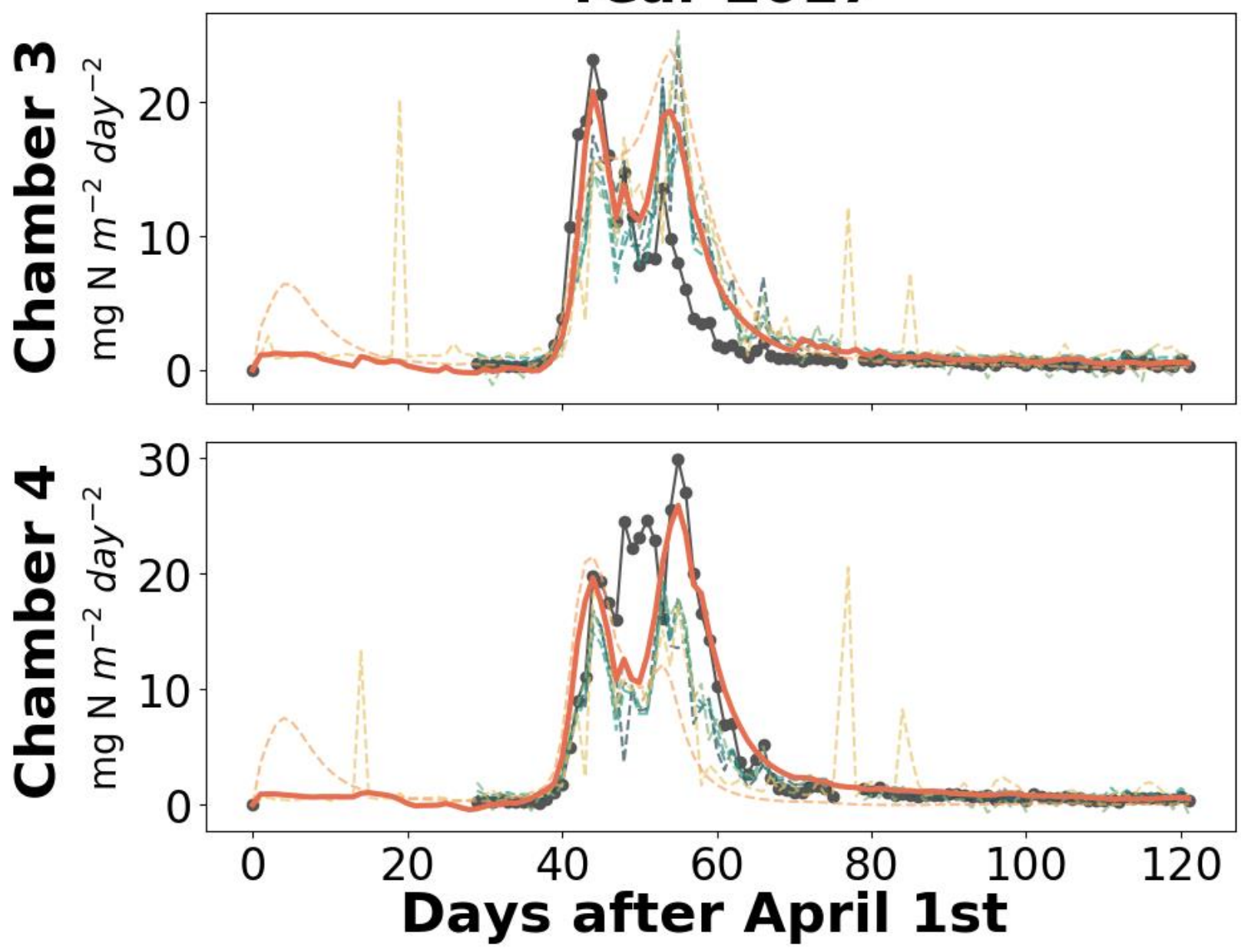

$$
\begin{aligned}
& \rightarrow \text { Obs, } N_{2} O \\
& \cdots \text { DT: } r_{U}^{2}=0.69, r_{L}^{2}=0.81 \\
& \cdots \text { RF: } r_{U}^{2}=0.65, r_{L}^{2}=0.85 \\
& \cdots \text { GB: } r_{U}^{2}=0.67, r_{L}^{2}=0.85
\end{aligned}
$$

$\mathrm{XGB}: r_{U}^{2}=0.57, r_{L}^{2}=0.87$

ANN: $r_{U}^{2}=0.54, r_{L}^{2}=0.76$

GRU: $r_{U}^{2}=0.60, r_{L}^{2}=0.57$

KGML-ag1: $r_{U}^{2}=0.78, r_{L}^{2}=0.86$

Figure S8: $\mathrm{N}_{2} \mathrm{O}$ flux time series comparisons between KGML-ag1 predictions (red solid line), pure ML models (other colored dashed line) and observations (black-dot line) from cross-validation on two representative panels of chamber 3 and 4 in 2016. The $\mathbf{r}^{2}$ value was calculated between observations and model simulations. $\mathbf{r}_{U}^{2}$ represents the $\mathbf{r}^{2}$ value from upper panel (chamber 3$)$ and $\mathbf{r}_{L}^{2}$ represents the $\mathbf{r}^{2}$ value from lower panel (chamber 4 ). 


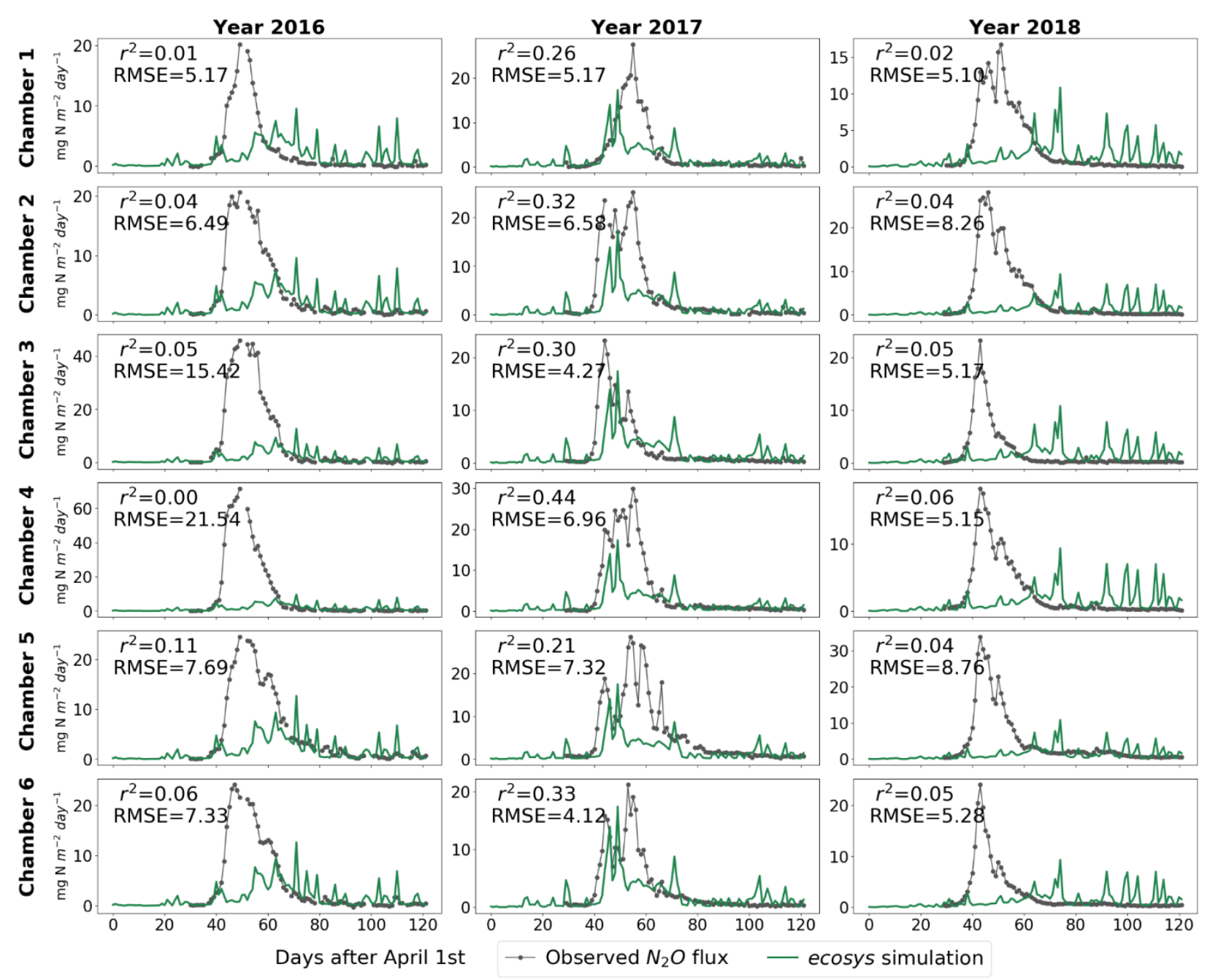

Figure S9: $\mathrm{N}_{2} \mathrm{O}$ flux time series comparisons between ecosys simulations (green line) and observations (black-dot line). 


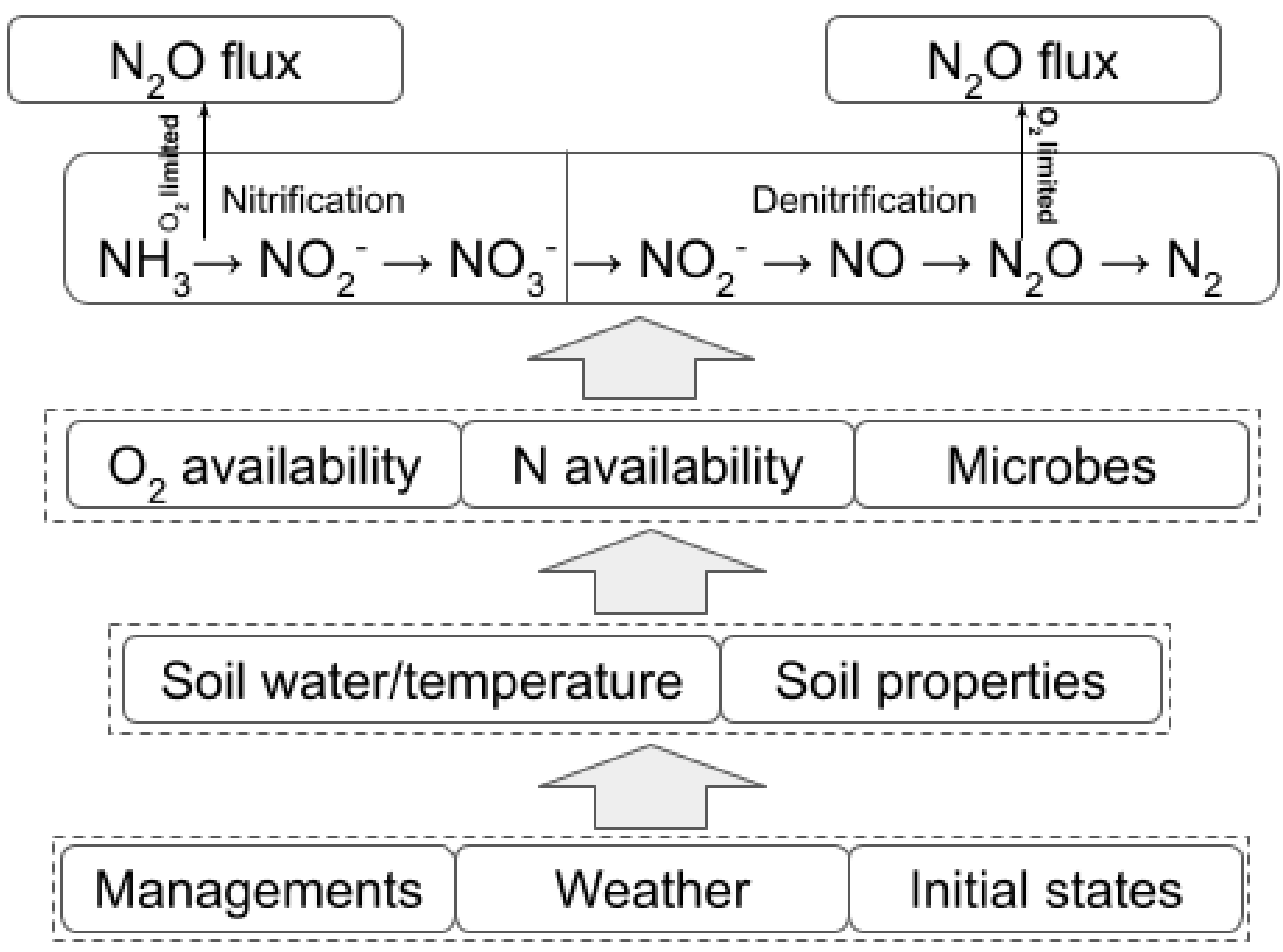

Figure S10: The simplified schema of $\mathrm{N}_{2} \mathrm{O}$ flux related variables and processes. 
Table S1: Variable short abbreviation, category (IMV represents intermediate variable, W represents weather forcing, FN represents the $\mathbf{N}$ fertilizer rate, SCP represents soil/crop property and target variable), description and units.

\begin{tabular}{|c|c|c|c|c|}
\hline No. & Abbreviation & Variable categary & Descriptions & Units \\
\hline 1 & RESIDUE_C & IMV & Total residue $\mathrm{C}$ on soil surface and in soil profile & $\mathrm{g} \mathrm{C} \mathrm{m}^{-2}$ \\
\hline 2 & HUMUS_C & IMV & Total particulate + non-particulate $\mathrm{C}$ in soil profile & $\mathrm{g} \mathrm{C} \mathrm{m}^{-2}$ \\
\hline 3 & LITTER_C & IMV & $\mathrm{C}$ in above + below-ground litterfall & $\mathrm{g} \mathrm{C} \mathrm{m}^{-2}$ \\
\hline 4 & CO2_FLUX & IMV & $\mathrm{CO}_{2}$ flux at the soil surface & $\mathrm{g} \mathrm{C} \mathrm{m}^{-2}$ day $^{-1}$ \\
\hline 5 & O2_FLUX & IMV & $\mathrm{O}_{2}$ flux at the soil surface & $\mathrm{g} \mathrm{O}_{2} \mathrm{~m}^{-2}$ day $^{-1}$ \\
\hline 6 & AUTO_RESP & IMV & Below-ground autotrophic (root) respiration & $\mathrm{g} \mathrm{C} \mathrm{m}^{-2}$ day $^{-1}$ \\
\hline 7 & MICRO_C & IMV & Microbial $\mathrm{C}$ in all residue and humus complexes & $\mathrm{g} \mathrm{C} \mathrm{m}^{-2}$ \\
\hline 8 & SURF_RES & IMV & Residue $\mathrm{C}$ on soil surface and in soil profile & $\mathrm{g} \mathrm{C} \mathrm{m}^{-2}$ \\
\hline 9 & CH4_FLUX & IMV & $\mathrm{CH}_{4}$ flux at the soil surface & $\mathrm{g} \mathrm{C} \mathrm{m}^{-2}$ day $^{-1}$ \\
\hline 10 & SURF_DOC_FLUX & IMV & $\begin{array}{l}\text { Flux of organic } \mathrm{C} \text { across all external surface boundaries in } \\
\text { runoff and sediment }\end{array}$ & $\mathrm{g} \mathrm{C} \mathrm{m}^{-2}$ day $^{-1}$ \\
\hline 11 & SUBS_DOC_FLUX & IMV & $\begin{array}{l}\text { Flux of organic } \mathrm{C} \text { across all external subsurface boundaries } \\
\text { in water dischage }\end{array}$ & $\mathrm{g} \mathrm{C} \mathrm{m}^{-2}$ day $^{-1}$ \\
\hline 12 & SURF_DIC_FLUX & IMV & $\begin{array}{l}\text { Flux of inorganic } \mathrm{C} \text { across all external surface boundaries in } \\
\text { runoff and sediment }\end{array}$ & $\mathrm{g} \mathrm{C} \mathrm{m}^{-2}$ day $^{-1}$ \\
\hline 13 & SUBS_DIC_FLUX & IMV & $\begin{array}{l}\text { Flux of inorganic } \mathrm{C} \text { across all external subsurface } \\
\text { boundaries in water dischage }\end{array}$ & $\mathrm{g} \mathrm{C} \mathrm{m}^{-2}$ day $^{-1}$ \\
\hline 14 & NBP & IMV & Net biome productivity & $\mathrm{g} \mathrm{C} \mathrm{m}^{-2}$ day $^{-1}$ \\
\hline 15 & $\mathrm{SOC}_{-} 1$ & IMV & Residue + humus $C$ in soil layer $1,5 \mathrm{~cm}$ depth & $\mathrm{g} \mathrm{C} \mathrm{m}^{-2}$ \\
\hline 16 & $\mathrm{SOC} \_3$ & IMV & Residue + humus $\mathrm{C}$ in soil layer $3,15 \mathrm{~cm}$ depth & $\mathrm{g} \mathrm{C} \mathrm{m}^{-2}$ \\
\hline 17 & SOC_5 & IMV & Residue + humus $\mathrm{C}$ in soil layer $5,28 \mathrm{~cm}$ depth & $\mathrm{g} \mathrm{C} \mathrm{m}^{-2}$ \\
\hline 18 & H2_FLUX & IMV & $\mathrm{H}_{2}$ flux at the soil surface & $\mathrm{g} \mathrm{H}_{2} \mathrm{~m}^{-2}$ day $^{-1}$ \\
\hline 19 & ECO_HVST_C & IMV & $\mathrm{C}$ removed in harvest & $\mathrm{g} \mathrm{C} \mathrm{m}^{-2}$ \\
\hline 20 & ECO_LAI & IMV & Leaf area index & $\mathrm{m}^{2} \mathrm{~m}^{-2}$ \\
\hline 21 & ECO_GPP & IMV & Gross primary productivity & $\mathrm{g} \mathrm{C} \mathrm{m}^{-2}$ day $^{-1}$ \\
\hline 22 & ECO_RA & IMV & Autotrophic respiration & $\mathrm{g} \mathrm{C} \mathrm{m}^{-2}$ day $^{-1}$ \\
\hline 23 & ECO_NPP & IMV & Net primary productivity & $\mathrm{g} \mathrm{C} \mathrm{m}^{-2} \mathrm{day}^{-1}$ \\
\hline 24 & ECO_RH & IMV & Heterotrophic respiration & $\mathrm{g} \mathrm{C} \mathrm{m}^{-2}$ day $^{-1}$ \\
\hline 25 & TTL_DIC & IMV & Total stocks of dissolved inorganic $\mathrm{C}$ & $\mathrm{g} \mathrm{C} \mathrm{m}^{-2}$ \\
\hline 26 & ET & IMV & Evapotranspiration rate & $m m$ day $^{-1}$ \\
\hline 27 & RUNOFF & IMV & Overland surface flow & $\mathrm{mm} \mathrm{day}^{-1}$ \\
\hline 28 & WATER & IMV & $\begin{array}{l}\text { The total amount of water in the rooting zone of the soil } \\
\text { profile }\end{array}$ & mm day ${ }^{-1}$ \\
\hline 29 & DISCHG & IMV & Water discharge flux through all subsurface boundaries & $\mathrm{mm}$ \\
\hline 30 & SNOWPACK & IMV & $\begin{array}{l}\text { The equivalent water content of snow }+ \text { ice }+ \text { water in the } \\
\text { snowpack }\end{array}$ & $\mathrm{mm}$ \\
\hline 31 & VWC_1 & IMV & The volumetric water content in soil layer $1,5 \mathrm{~cm}$ depth & $\mathrm{m}^{3} \mathrm{~m}^{-3}$ \\
\hline 32 & VWC_3 & IMV & The volumetric water content in soil layer $3,15 \mathrm{~cm}$ depth & $\mathrm{m}^{3} \mathrm{~m}^{-3}$ \\
\hline 33 & VWC_5 & IMV & The volumetric water content in soil layer $5,28 \mathrm{~cm}$ depth & $\mathrm{m}^{3} \mathrm{~m}^{-3}$ \\
\hline
\end{tabular}




SURF_WTR
ICE_1
ICE_2
ICE_3
PSI_1
PSI_3
PSI_5
WTR_TBL
RESIDUE_N
HUMUS_N
FERTZR_N
NET_PL_EXCH_N
NH4
NO3
SURF_DON_FLUX
SUBS_DON_FLUX
SURF_DIN_FLUX
SUBS_DIN_FLUX

IMV

FN

IMV

IMV

IMV

IMV

IMV

IMV

IMV

N2O_FLUX
NH3_FLUX
N2_FIXN

(

MICRO_N

NH4_1

NH4_3

NH4_5

NO3_1

NO3_3

NO3_5

NH4_RES

NO3_RES

ECO_HVST_N

N2_FLUX

RADN

TMAX_AIR

TDIF_AIR

HMAX_AIR

W
Near surface volumetric water content

The volumetric ice content in soil layer $1,5 \mathrm{~cm}$ depth

The volumetric ice content in soil layer $3,15 \mathrm{~cm}$ depth

The volumetric icecontent in soil layer $5,28 \mathrm{~cm}$ depth

The matric water potential in soil layer $1,5 \mathrm{~cm}$ depth

The matric water potential in soil layer $3,15 \mathrm{~cm}$ depth

The matric water potential in soil layer $5,28 \mathrm{~cm}$ depth

Depth of the water table from the surface

Total residue $\mathrm{N}$ on soil surface and in soil profile

Total particulate + non-particulate $\mathrm{N}$ in soil profile

$\mathrm{N}$ fertilizer applied

Net $\mathrm{N}$ exchange between soil and plants

Total $\mathrm{NH}_{4}{ }^{+}+\mathrm{NH}_{3}$ in the soil profile

Total $\mathrm{NO}_{3}{ }^{-}$in soil profile

$\mathrm{m}^{3} \mathrm{~m}^{-3}$

$\mathrm{m}^{3} \mathrm{~m}^{-3}$

$\mathrm{m}^{3} \mathrm{~m}^{-3}$

$\mathrm{m}^{3} \mathrm{~m}^{-3}$

Mpa

Mpa

Mpa

$\mathrm{m}$

$\mathrm{g} \mathrm{N} \mathrm{m}^{-2}$

$\mathrm{g} \mathrm{N} \mathrm{m}^{-2}$

$\mathrm{g} \mathrm{N} \mathrm{m}^{-2}$

$\mathrm{g} \mathrm{N} \mathrm{m}^{-2}$ day $^{-1}$

$\mathrm{g} \mathrm{N} \mathrm{m}^{-2}$

$\mathrm{g} \mathrm{N} \mathrm{m}^{-2}$

Flux of organic $\mathrm{N}$ across all external surface boundaries in $\mathrm{g} \mathrm{N} \mathrm{m}^{-2}$ day $^{-1}$ runoff and sediment

Flux of organic $\mathrm{N}$ across all external subsurface boundaries $\mathrm{g} \mathrm{N} \mathrm{m}^{-2} \mathrm{day}^{-1}$ in water dischage

Flux of inorganic $\mathrm{N}$ across all external surface boundaries in $\mathrm{N} \mathrm{m}^{-2}$ day $^{-1}$ runoff and sediment

Flux of inorganic $\mathrm{N}$ across all external subsurface $\quad \mathrm{g} \mathrm{N} \mathrm{m}^{-2}$ day $^{-1}$ boundaries in water dischage

Target variable $\mathrm{N}_{2} \mathrm{O}$ flux at the soil surface

$\mathrm{g} \mathrm{N} \mathrm{m}^{-2}$ day $^{-1}$

IMV $\quad \mathrm{NH}_{3}$ flux at soil and plant surfaces

$\mathrm{g} \mathrm{N} \mathrm{m}^{-2}$ day $^{-1}$

IMV Aerobic + anaerobic non-symbiotic $\mathrm{N}_{2}$ fixation + symbiotic $\mathrm{g} \mathrm{N} \mathrm{m}^{-2}$ day $^{-1}$ $\mathrm{N}_{2}$ fixation

IMV Total microbial $\mathrm{N}$ in all residue and humus complexes $\quad \mathrm{g} \mathrm{N} \mathrm{m}^{-2}$

IMV Total $\mathrm{NH}_{4}++\mathrm{NH}_{3}$ concentration in soil layer 1, 5cm depth $\mathrm{g} \mathrm{N} \mathrm{m}^{-2}$

IMV Total $\mathrm{NH}_{4}{ }^{+}+\mathrm{NH}_{3}$ concentration in soil layer 3, $15 \mathrm{~cm}$ depth $\mathrm{g} \mathrm{N} \mathrm{m}^{-2}$

IMV Total $\mathrm{NH}_{4}{ }^{+}+\mathrm{NH}_{3}$ concentration in soil layer 5, 28cm depth $\mathrm{g} \mathrm{N} \mathrm{m}^{-2}$

IMV Total $\mathrm{NO}_{3}{ }^{-}+\mathrm{NO}_{2}{ }^{-}$concentration in soil layer $1,5 \mathrm{~cm}$ depth $\mathrm{g} \mathrm{N} \mathrm{m}^{-2}$

IMV Total $\mathrm{NO}_{3}{ }^{-}+\mathrm{NO}_{2}{ }^{-}$concentration in soil layer $3,15 \mathrm{~cm}$ depth $\mathrm{g} \mathrm{N} \mathrm{m}^{-2}$

IMV Total $\mathrm{NO}_{3}{ }^{-}+\mathrm{NO}_{2}{ }^{-}$concentration in soil layer $5,28 \mathrm{~cm}$ depth $\mathrm{g} \mathrm{N} \mathrm{m}^{-2}$

IMV Residue $\mathrm{NH}_{4}{ }^{+}+\mathrm{NH}_{3}$ on soil surface and in soil profile $\mathrm{g} \mathrm{N} \mathrm{m}^{-2}$

IMV Residue $\mathrm{NO}_{3}{ }^{-}+\mathrm{NO}_{2}{ }^{-}$on soil surface and in soil profile $\quad \mathrm{g} \mathrm{N} \mathrm{m}^{-2}$

IMV N removed in harvest

IMV $\quad \mathrm{N}_{2}$ flux at the soil surface

Solar Radiation

$\mathrm{g} \mathrm{N} \mathrm{m}^{-2}$ day $^{-1}$

$\mathrm{g} \mathrm{N} \mathrm{m}^{-2}$ day $^{-1}$

Max air temperature

$\mathrm{W} \mathrm{m}^{-2}$

Difference between max and min air temperature

${ }^{\circ} \mathrm{C}$

${ }^{\circ} \mathrm{C}$

Max humidity fraction 


\begin{tabular}{|c|c|c|c|c|}
\hline 70 & HDIF_AIR & $\mathrm{W}$ & Difference between max and min humidity & fraction \\
\hline 71 & WIND & $\mathrm{W}$ & Wind speed & $\mathrm{m} \mathrm{s}^{-1}$ \\
\hline 72 & PRECN & $\mathrm{W}$ & Precipitation & $\mathrm{mm}_{\text {day }}{ }^{-1}$ \\
\hline 73 & TMAX_SOIL_1 & IMV & The maximum temperature in soil layer $1,5 \mathrm{~cm}$ depth & ${ }^{\circ} \mathrm{C}$ \\
\hline 74 & TDIF_SOIL_1 & IMV & $\begin{array}{l}\text { The difference between max and min temperature } \\
\text { temperature in soil layer } 1,5 \mathrm{~cm} \text { depth }\end{array}$ & ${ }^{\circ} \mathrm{C}$ \\
\hline 75 & TMAX_SOIL_3 & IMV & The maximum temperature in soil layer $3,15 \mathrm{~cm}$ depth & ${ }^{\circ} \mathrm{C}$ \\
\hline 76 & TDIF_SOIL_3 & IMV & $\begin{array}{l}\text { The difference between max and min temperature } \\
\text { temperature in soil layer } 3,15 \mathrm{~cm} \text { depth }\end{array}$ & ${ }^{\circ} \mathrm{C}$ \\
\hline 77 & TMAX_SOIL_5 & IMV & The maximum temperature in soil layer $5,28 \mathrm{~cm}$ depth & ${ }^{\circ} \mathrm{C}$ \\
\hline 78 & TDIF_SOIL_5 & IMV & $\begin{array}{l}\text { The difference between max and min temperature } \\
\text { temperature in soil layer } 5,28 \mathrm{~cm} \text { depth }\end{array}$ & ${ }^{\circ} \mathrm{C}$ \\
\hline 79 & TMAX_LITTER & IMV & The maximum temperature in litter & ${ }^{\circ} \mathrm{C}$ \\
\hline 80 & TDIF_LITTER & IMV & $\begin{array}{l}\text { The difference between max and min temperature } \\
\text { temperature in litter }\end{array}$ & ${ }^{\circ} \mathrm{C}$ \\
\hline 81 & ECND_1 & IMV & Electrical conductivity in soil layer $1,5 \mathrm{~cm}$ depth & $\mathrm{dS} \mathrm{m}^{-1}$ \\
\hline 82 & ECND_3 & IMV & Electrical conductivity in soil layer $3,15 \mathrm{~cm}$ depth & $\mathrm{dS} \mathrm{m}^{-1}$ \\
\hline 83 & ECND_5 & IMV & Electrical conductivity in soil layer $5,28 \mathrm{~cm}$ depth & $\mathrm{dS} \mathrm{m}^{-1}$ \\
\hline 84 & TTL_SALT_DISCHG & IMV & $\begin{array}{l}\text { Total salt discharge through water through all subsurface } \\
\text { boundaries }\end{array}$ & $\mathrm{g} \mathrm{Mg}^{-1}$ day $^{-1}$ \\
\hline 85 & PDOY & $\mathrm{SCP}$ & Plant day of the year & day \\
\hline 86 & CROPT & $\mathrm{SCP}$ & Crop type, 1 for corn and 0 for soybean & unitless \\
\hline 87 & TBKDS & $\mathrm{SCP}$ & Depth weighted averaged bulk density in soil profile & $\mathrm{Mg} \mathrm{m}^{-3}$ \\
\hline 88 & TCSAND & $\mathrm{SCP}$ & Depth weighted averaged sand content in soil profile & $\mathrm{g} \mathrm{kg}^{-1}$ \\
\hline 89 & TCSILT & $\mathrm{SCP}$ & Depth weighted averaged silt content in soil profile & $\mathrm{g} \mathrm{kg}^{-1}$ \\
\hline 90 & TPH & $\mathrm{SCP}$ & Depth weighted averagedpH in soil profile & unitless \\
\hline 91 & TCEC & $\mathrm{SCP}$ & Depth weighted averaged cmol+ $\mathrm{kg}-1$ in soil profile & $\mathrm{cmol}^{-1} \mathrm{~kg}^{-1}$ \\
\hline 92 & TSOC & SCP & Depth weighted averaged soil organic carbon in soil profile & $\mathrm{g} \mathrm{C} \mathrm{kg}^{-1}$ \\
\hline
\end{tabular}


Table S2: $\mathrm{N}_{2} \mathrm{O}$ prediction accuracy comparisons between LSTM and GRU models on synthetic data, with different combinations of IMVs (+9 or +58IMVs) and different sliding sliding window settings during training (e.g. 2y1y represent window size is 2 y ears and the window move 1 year after 1 iteration). Training Efficiency is also compared between LSTM and GRU models for the first two experiments, with changing the training counties $=3,10,30,70$, validation counties $=1,2,5,10$, and batch size $($ county numbers input in each iteration) $=1,5,5,5,5$.

\begin{tabular}{|c|c|c|c|c|c|c|}
\hline \multirow[b]{2}{*}{ Experiment settings } & \multicolumn{2}{|c|}{$\mathrm{N}_{2} \mathrm{O}$ prediction accuracy } & \multicolumn{4}{|c|}{ Training effeciency } \\
\hline & Test $\mathrm{r}^{2}$ & Test RMSE & $\begin{array}{c}\text { Train }=3, \text { val }=1, \\
\text { batch }=1\end{array}$ & $\begin{array}{c}\text { Train }=10, \text { val }=2, \\
\text { batch }=5\end{array}$ & $\begin{array}{c}\text { Train }=30, \text { val }=5, \\
\text { batch }=5\end{array}$ & $\begin{array}{c}\text { Train }=70, \text { val }=10, \\
\text { batch }=5\end{array}$ \\
\hline LSTM+9IMVs+1y1y & 0.74 & 1.32 & $3.8 \mathrm{~s}$ & $3.3 \mathrm{~s}$ & $9.2 \mathrm{~s}$ & $22 \mathrm{~s}$ \\
\hline GRU+9IMVs+1y1y & 0.81 & 1.08 & $3.5 \mathrm{~s}$ & $2.7 \mathrm{~s}$ & $7.2 \mathrm{~s}$ & $17 \mathrm{~s}$ \\
\hline LSTM+58IMVs+1y1y & 0.91 & 0.6 & & & & \\
\hline GRU+58IMVs+1y $1 \mathrm{y}$ & 0.92 & 0.59 & & & & \\
\hline LSTM+58IMVs+2y2y & 0.86 & 0.76 & & & & \\
\hline GRU+58IMVs+2y2y & 0.9 & 0.66 & & & & \\
\hline LSTM+58IMVs+2y1y & 0.89 & 0.67 & & & & \\
\hline GRU+58IMVs+2y $1 \mathrm{y}$ & 0.91 & 0.6 & & & & \\
\hline
\end{tabular}

